Revista lus et Praxis, Año 21, No 2, 2015, pp. 481 - 498

ISSN 0717 - 2877

Universidad de Talca - Facultad de Ciencias Jurídicas y Sociales

Ética de las creencias y defensa legal

Hugo Omar Seleme

Trabajo recibido el 4 de abril y aprobado el 30 de junio de 2015

\title{
Ética de las creencias y defensa legal
}

Hugo Omar Seleme*

\section{RESUMEN}

La ética legal ha estado concentrada en evaluar las acciones y las actitudes no proposicionales de los abogados. En el primer caso, ha establecido un catálogo de deberes y, en el segundo, una lista de virtudes. Este trabajo aborda el tema de la evaluación moral de las actitudes proposicionales, específicamente las creencias.

El trabajo explora la relevancia de los valores políticos para evaluar las creencias que los abogados defensores tienen con relación a sus clientes. El mismo presenta una concepción no-evidencialista según la cual los valores políticos -entendidos como algo diferente a las consideraciones epistémicas, prudenciales o de moral individual- son relevantes a la hora de evaluar dichas creencias.

ABSTRACT

The legal ethics has been focused on evaluating the actions and non-propositional attitudes of lawyers. In the first case, it has established a catalog of duties. In the second, it has identified a list of virtues.

This paper addresses the issue of the moral evaluation of propositional attitudes in particular of beliefs. The work explores the relevance of political values to evaluate the beliefs that defense lawyers have with regard to their clients. It presents a non-evidentialist conception according to which political values - understood as something different from epistemic, prudential or private moral considerations- are relevant to the assessment oflawyer's beliefs.

$$
\text { PALABRAS CLAVE }
$$

No-Evidencialismo, Actitudes no-proposicionales, Abogado Defensor

\section{KEYWORDS}

Non-Evidentialism, Non-propositional attitudes, Defense Lawyer

\section{Introducción}

Tradicionalmente, las consideraciones morales han sido relevantes para evaluar las acciones y las actitudes o virtudes de los abogados encargados de

\footnotetext{
* Investigador del CONICET, Argentina. Catedrático de Ética de la Facultad de Derecho de la Universidad Nacional de Córdoba. Profesor visitante de la Universidad Pompeu Fabra, del Heidelberg Center para América Latina, Universidad de Alcalá y el programa de doctorado de la Universidad de Chile. Director de la Maestría en Derecho y Argumentación de la UNC. Director del Programa de Ética y Teoría Política Universidad Nacional de Córdoba. Doctor en Derecho por la Universidad Nacional de Córdoba. Doctor en Derecho y Ciencias Sociales por la Universidad Nacional de Córdoba. Realizó instancias de investigación post-doctoral en el Princeton University Center for Human Values y Ohio University. Correo electrónico: hugoseleme@conicet.gov.ar.
} 
la defensa legal. No obstante, existe un aspecto del ejercicio de la defensa que la ética legal no ha explorado y que es el objeto del presente trabajo. Se trata de la evaluación moral de las creencias -y no sólo de las acciones o los rasgos de carácter- de quienes ejercen la defensa profesional. La pregunta que interesa abordar es la siguiente: ¿tiene el abogado defensor razones de moralidad política para poseer ciertas creencias en relación con su cliente?

El marco teórico en el que se articula el problema que pretendo abordar se conoce en filosofía moral como Ética de las Creencias. Esta rama de la filosofía moral se ocupa de las normas -epistémicas, prudenciales y morales- que gobiernan nuestra formación de creencias. Desde que William Kingdom Clifford publicase su trabajo seminal "The Ethics of Beliefs" ${ }^{11}$, un intenso debate ha tenido lugar acerca del tipo de requerimientos morales que se aplican a las creencias². Mientras Clifford defendía lo que luego pasó a conocerse como "Evidencialismo" -la idea de que es moralmente incorrecto creer algo cuando no se tiene evidencia suficiente-quien primero reaccionó a su artículo -William James- defendió una variante pragmatista de No-Evidencialismo. En "The Will to Believe" James sostenía que en algunos contextos es permisible y hasta obligatorio tener ciertas creencias aun si no existe suficiente evidencia que las respalden ${ }^{3}$.

Una de las aristas del debate ha estado referida al tipo de valores o normas que tienen relevancia a la hora de formar, mantener $u$ abandonar nuestras creencias. Algunos -siguiendo a Clifford- han señalado que lo relevante son los valores epistémicos; otros han defendido la relevancia de los valores morales, y un tercer grupo -siguiendo a James- ha puesto el énfasis en consideraciones prudenciales. El presente trabajo explora un aspecto novedoso y desatendido: la relevancia de los valores políticos. El mismo presenta una concepción noevidencialista según la cual los valores políticos -entendidos como algo diferente a las consideraciones epistémicas, prudenciales o de moral individual- son relevantes para evaluar la formación de creencias de los abogados defensores.

La mayor parte de la ética legal ha estado concentrada en evaluar las acciones y las actitudes no proposicionales de los abogados. En el primer caso han establecido un catálogo de deberes y en el segundo una lista de virtudes. El presente trabajo se vincula con la evaluación moral de las actitudes proposicionales, específicamente las creencias. ¿Tienen los abogados

\footnotetext{
${ }^{1}$ Ver ClifFord (1999), pp. 70-96.

${ }^{2}$ Las reflexiones sobre la ética de las creencias, sin embargo, no comenzaron con CLIFFORD. John LockE, al discutir el problema de la Fe en su An Essay Concerning Human Understanding, ya había defendido una posición evidencialista semejante a la de Clifford. Locke asentaba su variante moralista de evidencialismo sobre su concepción metaética del mandato divino. Ver Locke (1975).

${ }^{3}$ Ver JAMES (1979), pp. 291-341.
} 
razones de moralidad política para albergar ciertas creencias en relación con sus clientes?

\section{El problema}

Para visualizar el problema que pretendo abordar puede ser de utilidad presentar una situación hipotética. Imagine que $U d$. ha sido acusado de robar y está enfrentando un juicio. Un abogado se ha encargado de su defensa y ha argumentado celosamente por su inocencia. $U d$. le ha repetido desde que lo contrató que, más allá de la evidencia en su contra, Ud. es inocente.

El abogado tiene que preparar los alegatos finales y para eso lo ha citado en su lujoso despacho. A Ud. siempre le han Ilamado la atención unas pequeñas figuras de porcelana que tiene sobre el escritorio. Ud. está casi seguro de que se trata de porcelana china y en esta ocasión, como en las anteriores oportunidades que ha estado en el despacho, se detiene a verlas. Mientras Ud. conversa con su abogado ocasionalmente se distrae observando las figuras. $\mathrm{Ud}$. insiste en que ha sido acusado falsamente del robo. Ud. no es un ladrón. El abogado lo escucha tomando nota de sus dichos.

De repente una secretaria entra al despacho y le dice a su abogado que tiene una comunicación telefónica urgente que debe atender en la oficina contigua. El abogado se excusa por dejarlo solo unos instantes, pero gentilmente le explica que es una llamada impostergable. Antes de abandonar el despacho recoge todas las piezas de porcelana y las coloca en un cajón que cierra con llave.

Ud. se queda un poco aturdido por la escena, y piensa: “¿Por qué ha guardado las piezas? ¿Cree que se las voy a robar? ¿Acaso no le he dicho que no soy un ladrón? ¿Cree que soy culpable del robo a pesar de que está sosteniendo en el juicio que soy inocente?". Luego de que la primera oleada de preguntas ha pasado, Ud. se tranquiliza y piensa que tal vez ha guardado las piezas por otro motivo. Tal vez se trata de una acción mecánica que no tiene mayor significado. Una vez que regrese -se dice a sí mismo- le preguntará por lo sucedido y todo quedará aclarado.

$\mathrm{Ni}$ bien el abogado vuelve a la oficina, $U d$. le hace la pregunta que le ha estado dando vuelta por la cabeza. Le pide una explicación por su conducta. El abogado, un poco perplejo, responde: "Es algo que hago usualmente. Como Ud. imaginará en mi profesión no me cruzo con la gente más honesta del mundo, así que es lógico que tome mis recaudos". La respuesta lo deja un poco descolocado, después de todo $U d$. le ha dicho mil veces que es inocente y que no es un ladrón, así que vuelve a preguntarle: “¿Pero Ud. no creerá que soy un ladrón?". El abogado le recita una respuesta que parece haberse repetido a sí mismo muchas veces: "Lo que yo crea o no con relación a Ud. es irrelevante. Mi trabajo consiste en defenderlo, cuestionar la evidencia en su contra e intentar 
mostrar que su culpabilidad no está probada. Mi trabajo es encontrar defensas legales, no tener creencias".

¿Cómo se sentiría Ud. con esta respuesta? Creo que pocos clientes, que no hayan pasado por la facultad de derecho, se quedarían satisfechos. Si tuviesen la posibilidad de encontrar un abogado con igual pericia técnica pero que adicionalmente creyese en su inocencia, sin duda optarían por éste. Los clientes esperan que sus abogados no sólo hagan suyos sus intereses, defendiéndolos celosamente, sino que parecen exigir que los abogados muestren deferencia hacia sus dichos. No quieren sólo que el abogado con sus acciones vele por sus intereses. También esperan que sus creencias sean especialmente sensibles a sus afirmaciones.

Lo que el caso muestra es que el cliente pretende que el abogado le sea leal en diferentes aspectos. Un aspecto de la lealtad que debe existir entre abogado y cliente no es controvertido y ha sido muy explorado en la literatura especializada. Se trata de la lealtad en el interés o en la preocupación. El cliente espera que su abogado priorice sus intereses frente a los intereses de los demás. El cliente busca un abogado con capacidades técnicas porque da por sentado que éste las utilizará para promover sus intereses. Sin embargo, el ejemplo muestra que el cliente pretende que el abogado le sea leal en un aspecto menos estudiado y más controvertido. Pretende que el abogado le sea leal en sus creencias. Espera que el abogado esté especialmente inclinado a creer en su inocencia con independencia de la evidencia disponible, sólo porque él lo ha dicho. Pienso que no nos quedaríamos tranquilos si, puestos en la situación del ejemplo, nuestro abogado dijese: "Dada la evidencia disponible, y a pesar de que Ud. me dice que es inocente, no tengo más razones para creer que es inocente que las que dispongo para creer que es un ladrón. Ese es el motivo por el que tomo la precaución de guardar la porcelana. Sin embargo, defenderé su inocencia y protegeré sus intereses con todas mis herramientas técnicas".

En lo que sigue me detendré primero a especificar en qué consiste la exigencia de lealtad en las creencias que el cliente formula a su abogado (sección 3). A continuación exploraré las razones que existen para justificar las exigencias de lealtad en las creencias en un caso paradigmático: la amistad. Argumentaré para mostrar que el rasgo de las relaciones de amistad que hace surgir estas exigencias de lealtad se encuentra también presente en la relación abogado/ cliente (sección 4). Finalmente, me detendré a mostrar las razones de moralidad política que justifican las exigencias de lealtad en las creencias del abogado defensor (sección 5).

\section{Lealtad en las creencias}

Para entender en qué consiste la lealtad en las creencias puede ser de utilidad analizar un caso paradigmático de este tipo de lealtad: las relaciones de amistad. 
Supongamos que un amigo está acusado de cometer un delito, acusación que él niega. Si la evidencia en su contra es fuerte, pero no es concluyente, es seguro que tendremos una tendencia a creer en lo que dice nuestro amigo más allá de la evidencia ${ }^{4}$. Nuestro amigo espera que le creamos, no espera sólo que le digamos que le creemos o que defendamos sus intereses como si le creyésemos. Espera que estemos de su lado, no que finjamos estarlo ${ }^{5}$. Más aun, sentiríamos que hemos fallado como amigos si esto no sucediese y no nos conformaríamos con actuar como si le creyésemos.

Aunque se trata de un aspecto de la amistad que hasta hace poco había sido pasado por alto por los teóricos, los amigos esperan "something other than an impartial and disinterested review of the evidence where they are concerned" 6 . Un buen amigo "... does not defend her friend outwardly (which we earlier agreed a good friend would do) while inwardly believing the worst of her friend..." La amistad no sólo involucra la exigencia de ser leal a los intereses del amigo, sino también la de ser leal en las creencias. Los amigos no sólo son leales en sus acciones sino también en las opiniones o creencias que tienen del otro.

A pesar de que es indudable que la lealtad es un ingrediente de la amistad, conceptualizarla no ha sido una empresa pacífica. Algunas concepciones de la lealtad ponen especial acento en la promoción prioritaria de intereses. Philip Pettit, por ejemplo, afirma que ser leal a alguien equivale a estar "...dedicated to a particular individual's welfare..." ${ }^{\prime 8}$. Esta concepción de la lealtad es la que se encuentra presente en las concepciones tradicionales de la amistad. No obstante, pienso que se trata de una concepción equivocada de lealtad la que indefectiblemente conduce a que las concepciones de amistad que la adoptan sean igualmente defectuosas.

Este modo de concebir la lealtad es inadecuada porque, tal como señala Simon Keller, priorizar los intereses de un individuo no es ni necesario ni suficiente para mostrar lealtad hacia él ${ }^{9}$. No es suficiente porque uno puede priorizar los intereses de alguien por consideraciones distintas a las vinculadas a la lealtad, por ejemplo por consideraciones impersonales vinculadas con la justicia de su reclamo. Si pienso que entre dos reclamos conflictivos uno tiene más peso que el otro y decido priorizarlo, tal cosa no puede contar como una

\footnotetext{
${ }^{4}$ Ver Keller (2007), p. 27.

${ }^{5}$ Ver KelLer (2007), p. 31.

${ }^{6}$ Stroud (2006), p. 504.

7 Stroud (2006), p. 505.

8 Pettit (1988), p. 163.

${ }_{9}^{9}$ Ver Keller (2007), p. 9.
} 
muestra de lealtad. He priorizado los intereses de un individuo, pero no le he sido leal en ningún sentido.

Priorizar los intereses tampoco es necesario para que exista lealtad porque existen manifestaciones de lealtad que no están vinculadas con la promoción de intereses. Alguien es leal en relación con otro, por ejemplo, por identificarse con él. En esta manifestación de lealtad la persona leal "...treats the thing to which she is loyal as though it was her, feeling as she would feel and acting as she would act if certain things that are true of it were true of her..."10. Otra expresión de lealtad no vinculada con la promoción de intereses tiene que ver con la defensa de aquel a quien se debe lealtad. Si alguien está hablando mal de un amigo, por ejemplo señalando lo incompetente que es, la lealtad exige cuestionar lo que se está diciendo. Esto con total independencia de que esto no tenga ningún impacto en la promoción de sus intereses ${ }^{11}$. Otros modos de expresar lealtad están relacionados con la participación en rituales. Para una persona casada usar el anillo que intercambió con su pareja es una manifestación de lealtad, aunque esto no tenga ningún impacto en la promoción de los intereses del otro $^{12}$. Finalmente, la lealtad en las creencias es una manifestación de lealtad no vinculada con la promoción de intereses.

La lealtad a alguien es expresada como lealtad en las creencias si "...being loyal to X inclines you to hold or resist certain beliefs, independently of the evidence..."13. Este tipo de lealtad puede manifestarse de diferentes maneras. La primera manifestación se refiere a la forma en que procesamos los datos que se refieren a la persona que debemos lealtad. Stroud señala que "...we tend to devote more energy to defeating or minimizing the impact of unfavorable data than we otherwise would..."14. Si alguien afirma algo malo acerca del objeto de nuestra lealtad "...we are more likely to ask ourselves various questions about the person telling the story, the answers to which could discredit the evidence being presented... we will go to greater lengths... to construct and to entertain alternative and less damning interpretations of the reported conduct..."15. La segunda manifestación no tiene que ver con el proceso por el cual formamos nuestras creencias sino con los resultados. Cuando debemos a alguien lealtad hacemos en relación con ella diferentes inferencias y obtenemos diferentes conclusiones que las que obtendríamos si tal lealtad no existiese. No sólo

\footnotetext{
10 Keller (2007), p. 6.

11 Keller denomina a este tipo de lealtad "loyalty in advocacy".

12 Ver Keller (2007), p. 5.

13 Keller (2007), p. 6.

14 Stroud (2006), p. 505.

15 Stroud (2006), pp. 505-506.
} 
insumimos tiempo construyendo interpretaciones de sus acciones que sean más favorables, sino que tenemos la tendencia a creer estas explicaciones alternativas. Como consecuencia existe una probabilidad menor de que creamos que alguien a quien debemos lealtad hizo algo malo que la que existiría si no le debiésemos lealtad ${ }^{16}$.

Estas dos manifestaciones de la lealtad no hacen que los individuos se vuelvan ciegos a los hechos. La lealtad sólo produce que se tienda a interpretarlos de una manera diferente, más favorable a la persona a quien se es leal. Los recursos para lograr este objetivo son los que varían de una situación a otra. Stroud ha identificado los siguientes. En primer lugar, se puede intentar desacreditar la evidencia en contra de quien se tiene lealtad. En segundo lugar, pueden aceptarse los hechos pero interpretarlos de una manera que sea menos dañina. En tercer lugar, si la acción no puede reinterpretarse, entonces se la puede vincular a un rasgo de carácter valioso o virtuoso. En cuarto lugar, puede aceptarse que la evidencia es signo de un rasgo negativo en las acciones o el carácter de la persona, e intentar mostrar que estos rasgos forman parte de una virtud mayor. Finalmente, se puede reconocer la mala acción o el mal rasgo de carácter pero restarle importancia en la caracterización general de la persona ${ }^{17}$.

Para conceptualizar a la lealtad en las creencias no basta con enunciar sus manifestaciones y establecer los mecanismos que las provocan. Es necesario adicionalmente identificar sus elementos. Estos son tres. En primer lugar, la lealtad exige cierto vínculo emocional con la persona respecto a la cual se es leal. Debe existir una tendencia a albergar o resistir ciertas creencias en relación con quien se es leal "...that is not fully answerable to your rational or considered judgment..."18. Alguien espera que su amigo le crea cuando afirma su inocencia no meramente por consideraciones racionales o vinculadas con la evidencia disponible en el caso. Espera que crea en sus dichos con independencia de estas consideraciones. En segundo lugar, exige que lo que nos mueve a creer sean, en parte, consideraciones vinculadas con la persona respecto de la cual se es leal. Alguien espera que su amigo crea en sus dichos porque él ha dicho que es inocente. Espera que sus dichos por ser suyos tengan especial incidencia en las creencias de su amigo ${ }^{19}$. En tercer lugar, lo que nos mueve a creer tiene que ser la relación especial que existe con la persona a la que se es leal. Alguien

\footnotetext{
${ }^{16}$ Ver Stroud (2006), p. 506.

17 Ver Stroud (2006), p. 509.

${ }^{18}$ Keller (2007), p. 16.

${ }^{19}$ Ver Keller (2007), pp. 17-18.
} 
espera que su amigo crea en su inocencia no sólo porque es él quien afirma su inocencia, sino adicionalmente espera que le crea porque es $s u \mathrm{amigo}^{20}$.

\section{Lealtad en las creencias amistad y abogacía}

Nada de lo dicho hasta aquí sirve para justificar el deber de lealtad en las creencias que tienen los amigos y menos aún sirve para sostener que un deber semejante existe entre el abogado y su cliente. Los buenos amigos son leales en las creencias pero ¿qué justifica esta exigencia de lealtad? ¿Las razones que justifican esta exigencia se aplican también al caso del abogado defensor?

El aspecto característico de la amistad que justifica la exigencia de lealtad en las creencias que los amigos recíprocamente se deben, es el hecho de que el vínculo de amistad implica estar abierto a la influencia del otro. Ser amigo de alguien implica estar abierto a ser dirigido por las opiniones e intereses del otro y a ser interpretado por sus juicios ${ }^{21}$. A su vez, una persona está abierta a ser interpretada por las creencias que un amigo tiene de ella por el conocimiento íntimo que la amistad implica ${ }^{22}$. Un amigo es alguien que conoce todo de nosotros, es alguien que nos conoce mejor que nosotros mismos, de allí la importancia que otorgamos a sus opiniones sobre nuestra persona. Por último, el valor que es posible alcanzar en la amistad a través de este conocimiento

\footnotetext{
${ }^{20}$ Ver Keller (2007), pp. 18-20. Aunque Keller distingue la lealtad de interés y la lealtad en las creencias, parece suponer que de alguna manera se encuentran vinculadas. Así señala: "...when good friends form beliefs about each other, they sometimes respond to considerations that have to do with the needs and interests of their friends, not with aiming at the truth..." KeLleR (2007), p. 25. Hay dos maneras de interpretar este párrafo. O bien se entiende que los intereses que son promovidos por la lealtad en las creencias son de un tipo diferente de aquellos que son promovidos por la lealtad de interés o bien se entiende que son el mismo tipo de interés. Si se opta por lo primero, la lealtad en las creencias se distingue por lo que requiere -la formación y mantenimiento de creencias- y por el objetivo que persigue -promover ciertos intereses específicos-. Si se opta por lo segundo, la lealtad en las creencias se distingue sólo por lo que requiere. En todo otro sentido, permanece idéntica a la lealtad en interés.

${ }^{21}$ Esta visión de la amistad es presentada por Coking y Kennet. Ver Cocking y Kennett (1998), pp. 502527. Estar abierto a ser dirigido e interpretado por otro es el modo en que CockING y KeNETT conciben un rasgo esencial de la amistad: los amigos se preocupan y cuidan del otro. Que la amistad implica este cuidado y preocupación recíproca es aceptado casi unánimemente por quienes se han dedicado a su estudio. Ver Telfer (1970), pp. 223-241; Annas (1977), pp. 532-554; Friedman (1994); White (1999), pp. 19-34.

22 Este elemento de intimidad es puesto de manifiesto por Laurence Thomas. Según Thomas, que los amigos se confíen recíprocamente aspectos reservados y secretos de sus vidas es lo que ayuda a generar el vínculo de confianza que caracteriza a la amistad. Ver ThOmAs (1987), pp. 217-236. Esta tesis es independiente de la que sostiene que la intimidad es un elemento de la amistad. CockING y KENNETT tienen razón en rechazar la idea de que la confianza se genera por la intimidad. Ver CockING y KeNNETt (1998), p. 518. Sin embargo, no creo que a partir de esto pueda negarse que la intimidad es un elemento de la amistad.
} 
íntimo es el de "...to escape a little from your individuality, to reduce the intensity of being you..." 23

Puesto de otro modo, un valor que confieren los amigos a nuestra vida es el de la compañía. Este, por supuesto, no es el único valor de la amistad, pero indudablemente tener un amigo implica no estar solo, recluido en la propia individualidad. La amistad, en este sentido, relaja los límites y barreras que nos separan de los otros permitiendo que alguien entre a nuestro recinto más íntimo. Este conocimiento íntimo que los amigos poseen de nosotros nos vuelve especialmente receptivos a sus opiniones. Un amigo es quien nos conoce mejor que nosotros mismos, de allí la importancia que conferimos a las opiniones que tiene sobre nuestra persona. Esto, a su vez, pone a las personas en una posición de vulnerabilidad frente a sus amigos, lo que justifica las especiales exigencias vinculadas con la amistad. Entre éstas se encuentran las exigencias de lealtad en las creencias en sus diferentes manifestaciones.

La especial vulnerabilidad que una persona tiene frente a las opiniones que de ella posee un amigo es lo que justifica que los amigos deban evaluar la evidencia negativa que tienen en relación con la persona a quienes los une un lazo de amistad de un modo escrupuloso, minimizando su impacto, construyendo hipótesis explicativas más favorables. Esto también explica que las personas tengan el deber de someter a un escrutinio estricto a aquellas fuentes de información que arrojan datos negativos sobre quienes son sus amigos. Esta vulnerabilidad, adicionalmente explica el deber que tienen los amigos, no sólo de brindar interpretaciones alternativas de la evidencia contraria al amigo, sino también el deber de creerlas. Todas estas exigencias intentan prevenir el impacto que puede tener sobre la autoestima y la propia imagen de una persona el juicio negativo equivocado de aquel frente a quien ha relajado sus defensas, mostrándole su costado más íntimo.

Si esta apertura recíproca que los amigos hacen de su intimidad es lo que justifica las especiales exigencias de lealtad en las creencias que sobre ellos pesan, es posible inferir que sobre los abogados defensores pesan exigencias similares. Al menos esto es así en relación con los abogados que ejercen su profesión en el marco de sistemas acusatorios en los que el abogado es un celoso defensor de los intereses de su cliente. A semejanza de lo que sucede en la amistad, el abogado le debe a su cliente lealtad en los intereses. El abogado debe otorgar una prioridad especial a la promoción y defensa de los intereses de su cliente, a la defensa de su honor, su libertad, su vida y su propiedad. Esta idea del abogado como celoso defensor de los intereses de su cliente forma parte de lo que William H. Simon denomina "la visión dominante" del ejercicio

${ }^{23}$ Keller (2007), p. 38. 
profesional. Según este modo de concebir la abogacía, "the lawyer must-or at least may-pursue any goal of the client through any arguably legal course of action and assert any non frivolous legal claim" ${ }^{24}$.

A diferencia de lo que sucede en la amistad, sin embargo, la lealtad en los intereses del abogado con respecto a su cliente es unidireccional. El abogado debe lealtad a su cliente, pero esta exigencia de lealtad no es recíproca. El cliente no debe lealtad a los intereses de su abogado. Esta similitud parcial entre la relación abogado/cliente y los vínculos de amistad ha sido puesta de manifiesto por Charles Fried en su trabajo seminal "The Lawyer as a Friend"25. Para Fried, al igual que sucede con los amigos, los abogados pueden mostrar una especial preferencia por los intereses de su cliente sin que esto implique que están incumpliendo sus deberes morales generales, esto es, sin transformarse en malas personas.

Si el abogado va a llevar adelante la celosa defensa de los intereses de su cliente, característica del sistema acusatorio, necesita tener acceso a un cúmulo de información referida a su cliente. Mientras más información relevante para la causa posea el abogado, mayores son sus posibilidades de ejercitar una defensa exitosa. Sin embargo, como el juicio sobre qué información es relevante para la causa y cuál no sólo puede ser formulado desde el punto de vista experto del abogado, es necesario que el cliente ofrezca al abogado cualquier información, aun si de acuerdo con su criterio la posibilidad de que sea relevante para

${ }^{24}$ SimOn (1998), p. 7. Las Model Rules of Professional Conduct de la American Bar Association son un claro ejemplo de las reglas que regulan la profesión de abogado en un sistema adversarial. La regla 1.6 (a) establece que "(a) lawyer shall not reveal information relating to the representation of a client". Additionally the Model Rules recognizes that "(a) lawyer must also act with commitment and dedication to the interests of the client and with zeal in advocacy upon the client's behalf". (ABA Model Rules, art. 1.3, cmt.1). Este deber debe ser sopesado con el deber de candor establecido en la regla 3.3 (1): "A lawyer shall not knowingly make a false statement of fact or law to a tribunal or fail to correct a false statement of material fact or law previously made to the tribunal by the lawyer...". Con relación a los estándares de prueba, el privilegio abogado-cliente y el privilegio establecido en la quinta enmienda son especialmente importantes. Los Criminal Justice Standards de la ABA también son relevantes. El standard 4-7.6 establece que "...(t)he interrogation of all witnesses should be conducted fairly, objectively, and with due regard for the dignity and legitimate privacy of the witness, and without seeking to intimidate or humiliate the witness unnecessarily. Defense counsel's belief or knowledge that the witness is telling the truth does not preclude cross-examination...". El estandard 4-7.7 señala que "...in closing argument to the jury, defense counsel may argue all reasonable inferences from the evidence in the record. Defense counsel should not intentionally misstate the evidence or mislead the jury as to the inferences it may draw". Por ultimo, el standard 4-7.8 enfatiza que "...(d) Defense counsel should not intentionally refer to or argue on the basis of facts outside the record whether at trial or on appeal, unless such facts are matters of common public knowledge based on ordinary human experience or matters of which the court can take judicial notice".

${ }_{25}$ Ver Fried (1976), pp. 1060-1089. 
la defensa es mínima. El abogado es el que, luego de recibida la información, realizará una criba para determinar cuál es útil para la defensa y cuál no ${ }^{26}$.

$\mathrm{Al}$ igual que sucede en la amistad, en la relación abogado/cliente los límites y barreras que protegen la intimidad del cliente deben relajarse frente al abogado. Para que el abogado sea un celoso defensor de los intereses de su cliente, éste le permite ingresar al ámbito de su intimidad. Este conocimiento íntimo que el abogado posee del cliente hace que éste se vuelva especialmente receptivo y vulnerable a las opiniones de su abogado. La especial posición de vulnerabilidad en la que es puesto el cliente justifica las especiales exigencias que pesan sobre los abogados. El deber de lealtad en las creencias es una de estas exigencias.

El impacto que un juicio negativo equivocado puede tener sobre la propia imagen y la autoestima del cliente determina que el abogado esté sujeto a exigencias estrictas. Estas exigencias intentan minimizar el daño potencial que el cliente puede padecer. Sobre los abogados pesan exigencias de lealtad en las creencias análogas a las que se aplican en la amistad. La receptividad y vulnerabilidad del cliente a las opiniones de su abogado, dado el conocimiento íntimo que éste tiene de él, explica que a ambas relaciones humanas se apliquen exigencias semejantes.

Esto trae aparejado que, en primer lugar, el abogado a la hora de formar sus creencias debe evaluar la evidencia negativa que tienen en relación con su cliente de un modo escrupuloso, minimizando su impacto al construir hipótesis explicativas más favorables. Así, por ejemplo, si dada toda la evidencia disponible es igualmente probable que el cliente haya matado plenamente consciente o en un estado de emoción violenta, el abogado tiene razones para inclinarse por esta última explicación. Al igual que sucede en la amistad, no se trata de que el abogado tenga razones para actuar como si creyese tal cosa sino de que efectivamente la crea.

Adicionalmente, el abogado tiene razones para cuestionar aquellas fuentes de información que ofrecen datos negativos en relación con su cliente. Si un testigo, por ejemplo, señala que escuchó a su cliente planear premeditadamente

\footnotetext{
${ }^{26}$ La necesidad del abogado de tener acceso a toda la información posible por parte del cliente es generalmente utilizada para justificar el deber de confidencialidad. Este es un punto ulterior de semejanzas entre los deberes que surgen de la amistad y los que surgen de la relación abogado/cliente. JeREMY BeNTHAM y más recientemente DAVID LuBAN han desarrollado una crítica a esta justificación. Ver LUBAN (1988), pp. 189-191. Para quienes se sienten atraídos por esta línea de crítica todavía es posible suscribir lo que señalo en el texto sobre la cantidad de información a la que tiene acceso un abogado. Basta que compartan la idea de que para ejercitar una defensa legal eficiente el abogado necesita conocer información del cliente que se encuentra en su esfera de intimidad. La crítica de Benthan y Luban pone en duda la afirmación de que sin el deber de confidencialidad los clientes vayan a temer revelar información potencialmente relevante. Ambos, sin embargo, no cuestionan que para ejercitar la defensa es necesario que el abogado tenga acceso al mayor monto de información posible. Esto sólo basta para el argumento ofrecido en el texto.
} 
el homicidio, el abogado debe explorar todas las posibilidades de mostrar que tal testimonio es sesgado y poco creible. Una vez más, no se trata de que tenga razones para socavar la credibilidad del testigo frente al juez mientras él internamente cree lo contrario. Tiene razones para buscar información que socave la credibilidad del testigo frente a sus propios ojos.

Por último, cuando es imposible construir explicaciones alternativas más favorables al cliente en base a la evidencia disponible, y no es posible cuestionar la fuente de dicha evidencia, el abogado tiene razones para minimizar el impacto que este juicio negativo tiene sobre el juicio general que formula sobre la persona de su cliente. Si los hechos son incontrovertibles y de acuerdo a toda la evidencia el cliente mató de manera premeditada, el abogado tiene razones para no generalizar este juicio negativo a toda la persona del cliente. Específicamente, tiene razones para creer que, aunque mató, no "es un criminal". Su acción no es definitoria de su persona. Si bien es muestra de un rasgo de carácter negativo, el cliente posee otros rasgos de carácter positivo que impiden generalizar el juicio negativo a todos los aspectos de su persona.

Que las exigencias de lealtad en las creencias se apliquen a la relación abogado/cliente trae aparejado algunas consecuencias. Las razones que tiene un abogado para evaluar la evidencia y albergar o revisar creencias en relación con su cliente no son todas de índole epistémico. Algunas de ellas, las que surgen de la lealtad, están fundadas en la situación de vulnerabilidad en la que se encuentra el cliente. Es el tipo especial de vínculo que existe entre el abogado y el cliente lo que da lugar a estas razones. Así, por ejemplo, el abogado tiene razones para creerle al cliente que afirma su inocencia que van más allá de la evidencia disponible. Adicionalmente, el abogado tiene razones especiales para creer en la afirmación de inocencia de su cliente porque él ha dicho que es inocente. Los dichos de su cliente deben tener especial incidencia en sus creencias por ser suyos. Por último, el abogado tiene razones para creer en lo que afirma su cliente debido a la relación especial que mantiene con él. El abogado debe creer en su cliente porque es su cliente.

Lo señalado no implica que el abogado deba ser ciego a la evidencia disponible, o que no tenga razones epistémicas para albergar creencias. Simplemente implica que el vínculo abogado/cliente genera razones para creer que se suman a, y muchas veces entran en conflicto con las razones epistémicas.

Que la lealtad en las creencias no tenga como consecuencia la ceguera ante los hechos permite enfrentar una posible objeción a la idea de que el abogado debe este tipo de lealtad a su cliente. El problema reside en que esta lealtad impida que el abogado pueda llevar adelante una defensa adecuada debido a su incapacidad para evaluar de manera imparcial la evidencia rendida en la 
causa. Las creencias del abogado estarían sesgadas y su juicio sobre qué se encuentra probado y qué no estaría igualmente sesgado. Dado que lo que le interesa al cliente en última instancia es la decisión adoptada por el juez, y dado que este no le debe ninguna lealtad en las creencias, podría suceder que el abogado creyese falsamente que ha brindado elementos suficientes al juez para justificar un dictamen favorable a su cliente pero que lo creyese en parte debido al sesgo que posee a favor de su cliente.

Expresado técnicamente el problema es el siguiente. La lealtad en las creencias del abogado determina que éste aplique un estándar de prueba sesgado a favor de su cliente. El juez, en cambio, no posee este sesgo y aplica un estándar de prueba no sesgado. Como la función del abogado es ofrecer argumentos que sirvan para justificar una decisión judicial a favor de su cliente, el que utilice un estándar de prueba sesgado diferente al utilizado por el juez conspira en contra de que cumpla eficazmente su misión ${ }^{27}$.

Pienso que, más allá de las apariencias, el problema no es tan grave como parece. En primer lugar, que la lealtad en las creencias no provoque ceguera sobre los hechos hace perfectamente posible que el abogado sepa que el juzgador está utilizando un estándar de prueba diferente al suyo y que, por tanto, guíe su práctica defensiva a partir de este dato. En segundo lugar, en aquellos sistemas procesales que incorporan la presunción de inocencia el estándar de prueba del juzgador y el abogado no serán discrepantes. La existencia de la presunción sesga el estándar de prueba dentro del proceso de la misma manera que la lealtad en las creencias del abogado. Allí donde la presunción de inocencia está en pie, el estándar de prueba posee un sesgo no epistémico a favor del acusado. La prueba para condenar debe ser lo suficientemente fuerte como para derrotar la presunción de inocencia ${ }^{28}$.

\footnotetext{
27 Agradezco a un evaluador anónimo el haberme hecho notar la necesidad de enfrentar este problema.

${ }^{28}$ Es posible ofrecer una justificación del principio de inocencia en la misma concepción de legitimidad política que presento en la próxima sección. Aunque no puedo detenerme en este punto aquí, la idea básica es la siguiente. La coacción estatal está moralmente justificada cuando es ejercitada por una comunidad política donde cada ciudadano es tratado como un autor. Tratar a los ciudadanos como coautores de una empresa colectiva implica presumir que cada uno ha hecho su parte, es decir que cada uno ha cumplido las reglas que organizan la empresa colectiva. Por lo tanto, un sistema procesal que no incluye la regla de la presunción de inocencia -y el estándar de prueba correlativo- no trata a los ciudadanos como autores y no es apto para administrar la coacción de modo que se encuentre moralmente justificada.

El principio de inocencia, sin embargo, no exige que el juzgador deba creer en la inocencia del acusado. Se trata de una regla institucional que exige tratarlo como si fuese inocente, es decir, como si hubiese hecho su parte. La lealtad en las creencias, por el contrario, brinda razones para que el abogado tenga cierta opinión sobre su cliente. Para una discusión sobre los diferentes contextos a los que es aplicable el principio de inocencia, puede verse Duff (2013), pp. 170-192.
} 


\section{Autoría política y lealtad en las creencias}

El argumento que he ofrecido hasta aquí sostiene, en primer lugar, que existen rasgos de la relación de amistad que justifican la exigencia moral de lealtad en las creencias. Este rasgo es la vulnerabilidad que una persona tiene a la opinión de quien es su amigo. La vulnerabilidad, a su vez, se funda en el conocimiento íntimo que caracteriza a las relaciones de amistad. Sin este conocimiento recíproco el bien humano que ofrece la amistad -la compañía de otro que nos conoce mejor que nosotros mismos y cuida de nuestros intereses- no es posible de alcanzar. Si esto es así, es el vínculo de amistad y las razones morales que lo hacen bueno y valioso lo que justifica en última instancia la lealtad en las creencias que los amigos se deben.

En segundo lugar, el argumento ha mostrado que el cliente tiene en relación con las opiniones de su abogado un grado de vulnerabilidad semejante al que encontramos en la amistad. Al igual que sucede en la amistad la vulnerabilidad se debe al conocimiento íntimo que el abogado debe tener de su cliente para ejercitar la defensa. Sin este conocimiento la celosa defensa que el sistema acusatorio garantiza al cliente no podría ser llevada adelante. Es el vínculo abogado/ cliente característico del sistema acusatorio y las razones que lo vuelven valioso lo que justifica la lealtad en las creencias que el abogado debe a su cliente.

El argumento a favor de la lealtad en las creencias del abogado no estará completo hasta que no se hayan ofrecido las razones que justifican el sistema acusatorio y su peculiar modo, de configurar el rol del abogado defensor. Como no puede ser de otro modo, la justificación del sistema acusatorio depende de una teoría de la legitimidad política. Aunque no puedo detenerme en los detaIles aquí, me interesa poner de manifiesto la manera en que la concepción de legitimidad política que defiendo -a la que he denominado la legitimidad como autoría- ofrece razones para justificar el sistema acusatorio y, en última instancia, para justificar la lealtad en las creencias del abogado con relación a su cliente.

La idea básica de la legitimidad como autoría es la siguiente ${ }^{29}$. El ejercicio de la coacción a través de las instituciones jurídicas genera una amenaza para individuos que -en tanto agentes racionales- tienen interés en dirigir sus vidas a partir de sus propias consideraciones. La amenaza reside en que los cursos de acción que adoptan los individuos, los estados de cosas que soportan y hasta su misma configuración personal, esté determinada por las instituciones estatales en lugar de sus propias consideraciones. El riesgo reside en que el interés que tiene cada ciudadano -en tanto agente racional- en ser el autor de su propia vida y configuración personal se vea frustrado por la existencia de instituciones coercitivas.

${ }^{29}$ He expuesto esta concepción de legitimidad en Seleme (2010), pp. 73-99. 
El modo de conjurar esta amenaza, la respuesta al problema, consiste en hacer que los individuos a quienes se aplican coercitivamente las instituciones jurídicas sean colocados por ellas en el rol de autores ${ }^{30}$. Si en tanto agente sensible a razones un individuo tiene interés en dirigir su vida a partir de sus propias consideraciones, en ser su autor, y si el carácter coercitivo del esquema institucional puede conspirar en contra de la satisfacción de aquel interés, la solución es hacer que todos aquellos a quienes se aplica sean autores del mismo.

Un esquema institucional estatal es legítimo, por tanto, cuando todos aquellos a quienes establece como destinatarios son sus autores. Dicho de otro modo, un esquema es legítimo cuando satisface las condiciones que permiten imputar su autoría a todos aquellos a quienes se aplica. Lo que se requiere es que las instituciones estatales coloquen a los ciudadanos en un determinado rol: el de autor. La idea es que los sujetos a quienes se aplican las instituciones no son autores del diseño institucional porque lo configuren a través de su participación efectiva, sino que es el diseño institucional el que los configura como autores. Si un esquema institucional satisface los intereses que los sujetos a quienes se aplica poseen en tanto autores, entonces los transforma en tales. Puesto que el principal interés que -en tanto autores- poseen los ciudadanos en relación con sus instituciones es el de participar efectivamente en su diseño y configuración, si las instituciones posibilitan tal cosa, entonces son de su autoría, esto es, son legítimas.

Un esquema institucional no trata a los ciudadanos como autores cuando no permite que sus opiniones e intereses cuenten a la hora de tomar decisiones. Los trata como meros súbditos, receptores de órdenes, de quienes sólo se pretende obediencia. Siguiendo a Charles Beitz ${ }^{31}$ podemos denominar a este interés de autoría como interés en la responsabilidad deliberativa ${ }^{32}$.

El interés en la responsabilidad deliberativa se refiere a la posibilidad de participar en los procedimientos políticos de toma de decisión que se aplican coercitivamente a un individuo. El caso de las condenas penales es un ejemplo paradigmático de decisión coercitiva. El interés reside en que la toma de decisiones colectivas pueda hacerse en base a una deliberación pública suficientemente informada, donde las opiniones e intereses de los afectados puedan ser consideradas y evaluadas responsablemente. Este interés es satisfecho por el sistema acusatorio, toda vez que establece que el abogado defensor debe ser un celoso defensor de los intereses de su cliente y un vocero de sus opiniones.

\footnotetext{
${ }^{30}$ Lo señalado presupone que la existencia del Estado se encuentra justificada, tema que no he abordado en el presente trabajo, donde sólo me interesa el problema de la legitimidad.

31 Ver Beitz (1990).

32 Junto con el interés en la responsabilidad deliberativa existen otros dos intereses de autoría que no analizo en el texto: el interés en el modo de tratamiento y el interés en el reconocimiento.
} 
Un sistema que impide que los intereses o las opiniones del acusado sean escuchados no satisface el interés que todos los ciudadanos en tanto autores tienen en la responsabilidad deliberativa. En un sistema inquisitivo los ciudadanos son tratados como meros súbditos de quien se requiere sumisión, y no como autores que merecen ser escuchados y respetados.

El sistema acusatorio al garantizar que las opiniones y los intereses de los acusados sean tenidos en cuenta a la hora de dictar sentencia, ubica a los acusados en el rol de autores dentro del proceso penal. Esto a su vez hace que el acusado pueda ser visto como autor de la condena que se le impone. Finalmente, que el acusado sea el autor de la condena hace que la imposición de la misma se encuentre moralmente justificada.

Lo que he señalado en esta última sección es esquemático pero puede ayudar a ver la manera en que las razones de legitimidad política justifican que los abogados deban lealtad en las creencias en relación a sus clientes. El sistema acusatorio se encuentra justificado en la exigencia de tratar a los ciudadanos como autores de las decisiones que se les aplican de modo coercitivo. Los ciudadanos son tratados como autores cuando el entramado institucional permite, entre otras cosas, que sus opiniones e intereses cuenten. La exigencia propia del sistema acusatorio de que los abogados sean celosos defensores de los intereses de sus clientes está orientada a satisfacer este interés de autoría. Esta celosa defensa requiere que el abogado tenga acceso al ámbito de intimidad del cliente. Este acceso a la intimidad del cliente por parte del abogado lo vuelve especialmente vulnerable a sus opiniones, y es esta vulnerabilidad la que justifica las exigencias de lealtad en las creencias que pesan sobre el abogado. Por lo tanto, las razones que en última instancia justifican la lealtad en las creencias del abogado son de índole política y tienen que ver con la exigencia de que los ciudadanos sean tratados como autores.

Las razones de moralidad política que exigen que los ciudadanos sean tratados como autores justifican que los abogados deban a sus clientes lealtad en la promoción de sus intereses. Son estas mismas razones las que fundan la exigencia lealtad en las creencias. Se trata de razones que nada tienen que ver con la verdad de la creencia que el abogado alberga. Son razones políticas, no epistémicas.

\section{Bibliografía CitADA}

AnNAS, Julia (1977): Plato and Aristotle on Friendship and Altruism. Mind (Oxford, Oxford University Press), Vol. 86.

BeItz, Charles (1990): Political Equality (Princeton, New Jersey: Princeton University Press).

Clifford, W. K. (1999): "The Ethics of Belief", en: The Ethics of Belief and other Essays (Amherst MA, Prometheus). 
CoCKING, Dean y KenNett, Jeanette (1998): "Friendship and the Self", en: Ethics. № 108 (Chicago, University of Chicago Press).

Duff, Antony (2013): "Who Must Presume Whom to Be Innocent of What?", en: Netherlands Journal of Legal Philosophy (Vol. 42, № 3).

Fried, Charles (1976): "The Lawyer as a Friend: The Moral Foundations of the Lawyer/Client Relationship", en: Yale Law Journal (Vol. 85).

Friedman, Marilyn (1994): What are Friends for? Feminist Perspectives on Personal Relationships and Moral Theories. (Ithaca N.Y., Cornell University Press).

James, William (1979): "The Will to Believe", en: The will to believe and other essays in popular philosophy (Cambridge, Massachusetts: Harvard University Press).

KELLER, Simon (2007): The Limits of Loyalty (Cambridge, Cambridge University Press).

LOCKE, John (1975): An Essay Concerning Human Understanding (Oxford: Clarendon Press).

Luban, David (1988): Lawyers and Justice (Princeton, New Jersey: Princeton Unviersity Press).

Pettit, Philip (1988): "The Paradox of Loyalty", en: American Philosophical Quarterly (Vol. 25).

Seleme, Hugo Omar (2010): "La Legitimidad como Autoría", en: Revista Brasilera de Filosofía.

Simon, William H. (1998): The Practice of Justice: A Theory of Lawyer's Ethics (Cambridge, Massachusetts, Harvard University Press).

Stroud, Sarah (2006): "Epistemic Partiality in Frienship", en: Ethics. 2006 (Vol. 116), p. 504.

TelfER, Elizabeth (1970): "Friendship", en: Proceedings of the Aristotelian Society. ThOMAS, Laurence (1987): "Friendship", en: Synthese (Vol. 72).

WHITE, Richard (1999): "Friendship and Commitment", en: Journal of Value Inquiry (Vol. 39), pp. 19-34. 